\title{
Effectiveness of Patient Group Meetings for Postoperative Patients with Defecatory Dysfunction
}

\author{
Asami Tsuji ${ }^{1,2}$, Miyoko Nakayama ${ }^{3}$, Katsunari Takifuji ${ }^{4}$, Masako Yamaguchi $^{2}$, Itsuyo Ueda ${ }^{2}$, Ikuharu Morioka ${ }^{2}$, Yukiko \\ Suzuki ${ }^{5}$ and Kazuhisa Miyashita ${ }^{1}$ \\ ${ }^{1}$ School of Medicine, Wakayama Medical University, kimiidera 811-1, Wakayama, 641-8509, Japan \\ ${ }^{2}$ School of Health and Nursing Science, Wakayama Medical University, Mikazura 580, Wakayama, 641-0011, Japan \\ ${ }^{3}$ Wakayama Medical University Hospital, Wakayama, kimiidera 811-1, 641-8509, Japan \\ ${ }^{4}$ Saiseikai Arida Hospital, Yuasa, Arida, Wakayama 643-0007, Japan \\ ${ }^{5}$ School of Nursing, Shijonawate Gakuen University, Daito, Osaka 574-0001, Japan
}

\section{Abstract}

Background: Research on a peer support for patients after low anterior resection (LAR) is now limited. Little is then known about the effectiveness of patient group meeting for such patients. The aim of this study was to clarify the effectiveness of patient group meetings for the LAR postoperative patients with defecatory dysfunction.

Methods: A longitudinal prospective study was conducted. Twenty-six patients (mean age: 65.6 years old) participated in this study. The patient group meetings were held three times for six months, consisting health education and group discussions. Knowledge and Emotion were measured using visual analogue scale. Defecatory dysfunction was measured using a scale. The QOL was measured by EORTC QLQ-C30. T test, Chi-squared or Fisher's exact test, paired t test, multiple linear regression analysis and Spearman's rank correlation were used for the statistical analyses.

Results: Participants in the patient group meetings gained information on defecatory dysfunction and coping with diarrhea. Their score of scales estimating defecatory dysfunction were stable. They reported higher, but not significant, global health status/QOL and significantly higher Social Functioning. Significant decreased symptom scores of Fatigue and Diarrhea were obtained depending on the participation rate. A decreased symptom score in Diarrhea was related to the increase in the global health status/QOL and Social Functioning, and not related to the change in the defecatory dysfunction.

Conclusion: A patient group meeting for the patients after LAR has positive health effects on the QOL of diarrhea. The results suggest the importance of patient group meeting to survival related to QOL of such patients. Healthcare providers should be aware of the importance of patient group meeting to improve the survival related to QOL in the postoperative patients with defecatory dysfunction.

\section{Introduction}

In Japan, the number of colorectal cancer patients has drastically increased in recent years [1]. The rectum is the most common site for colorectal cancers to establish. Treatment for rectal cancer mainly involves surgery, which saves the external anal sphincter.

Low anterior resection (LAR) surgery is one of the major operations for rectal cancer [2]. Patients who have undergone LAR are likely to face complications, including defecatory dysfunction [3-5]. These are not only physically debilitating $[6,7]$ but also negatively effect quality of life (QOL) [8-10]. This negative effect on QOL for LAR postoperative patients is more deleterious than that in patients who have undergone colostomy [11]. Current support for LAR postoperative patients is mainly focused on managing defecatory dysfunction and establishing a regular lifestyle and proper eating habits. In the case of postoperative needs, nurses do not at this time fully provide adequate care for patient's QOL. Increased QOL is essential for the survival of the patient.

A previous report clarified that support for LAR postoperative patients with defecatory dysfunction should be continued until they are able to control such difficulties and, furthermore, that they be provided opportunities both to gain knowledge for managing defecatory dysfunction (educational support) and to interact with other patients suffering from the same disorder in order to mutually exchange information and share experiences (emotional support) [12].

This study aimed to clarify the effectiveness of patient group meetings including programs to define educational and emotional support for the LAR postoperative patients with defecatory dysfunction, and also to note findings with which to support patient's survival related to QOL.

\section{Subjects and Methods}

\section{Study design}

Longitudinal prospective design was used with a pre-test and a post-test. This study was held at a Japanese university hospital from January 2015 to October 2015. An overview of the entire design of this study is shown in Figure 1.

"Corresponding Author: Asami Tsuji, School of Health and Nursing Science, Wakayama Medical University, Mikazura 580, Wakayama, 641-0011, Japan; E-mail: tsuji-nc@wakayama-med.ac.jp

Citation: Tsuji A, Nakayama M, Takifuji K, Yamaguchi M, Ueda I, et al. (2017) Effectiveness of Patient Group Meetings for Postoperative Patients with Defecatory Dysfunction. Int J Nurs Clin Pract 4: 260. doi: https://doi. org/10.15344/2394-4978/2017/260

Copyright: () 2017 Tsuji et al. This is an open-access article distributed under the terms of the Creative Commons Attribution License, which permits unrestricted use, distribution, and reproduction in any medium, provided the original author and source are credited. 
Citation: Tsuji A, Nakayama M, Takifuji K, Yamaguchi M, Ueda I, et al. (2017) Effectiveness of Patient Group Meetings for Postoperative Patients with Defecatory Dysfunction. Int J Nurs Clin Pract 4: 260. doi: https://doi.org/10.15344/2394-4978/2017/260

Page 2 of 7

\section{Patient group meetings}

A specialist team, consisting of a colorectal surgeon, a chief nurse, a certified social insurance labor consultant and two researchers, was trained according to our patient group meeting protocols. The patient group meetings were held three times. The patients participated in the group meetings of their own free will and participation was free.

The same health provider participated in all three of these group meetings. The programs of each meeting were basically similar. Before the first meeting and after the first and the second meetings, the health providers shared the knowledge and ideas of the two core support programs, health education and group discussions.

The peer support has often been provided until now mainly for breast cancer patients $[13,14]$, and there are educational models for such patients [15]. An educational model for rectal cancer patients has not been established in Japan as yet. Health education is based on an educational model that was developed by Fawzy et al. $[16,17]$ and which has been adapted by Fukui for Japanese breast cancer patients $[18,19]$. Our 90-minute health education program included teaching of relevant topics to increase knowledge for solving problems encountered in daily life. Based on available literature [12], four educational theme were set; knowledge on defecatory dysfunction, coping strategy, cancer and working. The specific topics were then colorectal cancer, operation and mechanism of defecatory function (First Meeting), palliative care (Second Meeting) and self-care and working life (Third Meeting).

The 90-minute group discussions focused on sharing disquieting concerns of metastasis or reoccurrence of cancer with other patients who were coping with the same disease. Four emotional supports were dedicated; fear associated with cancer, feelings of loneliness, feelings of relief, and confidence in daily life. The participants were divided into several groups consisting of six to seven persons. One of the health providers served as a facilitator for the participants to share information on symptoms or detailed methods to decrease them, and to express anxieties and expectations. The make up of the groups was not set and the participants freely formed the groups themselves.

\section{Participants}

The participants of the study consisted of patients who had undergone LAR from 2011 to 2014. Participants were limited to those whose general condition after the operation was good, who had no metastasis and no recurrence of the cancer, and who had no psychosomatic symptoms due to chemotherapy or radiation therapy. If the patients had undergone diverting stoma, they were enrolled after the diverting stoma was closed. Among them, only those who had received a comprehensive diagnosis by the colorectal surgeon and who wished to participate in the study of their own free will were selected.

The colorectal surgeon and the chief nurse selected 84 patients from the patient list of the hospital under these criteria, and sent out letters of invitation with a detailed explanation of the study (Figure 1). Fortyone patients returned the letter and thirty showed the agreement (participant rate $35.7 \%$ ). In this study, 26 participants who had responded to both the pre-test and the post-test served as the subjects.

\section{Measurements}

Demographic data, details regarding knowledge and emotion, defecatory dysfunction, and QOL were collected from self-reported

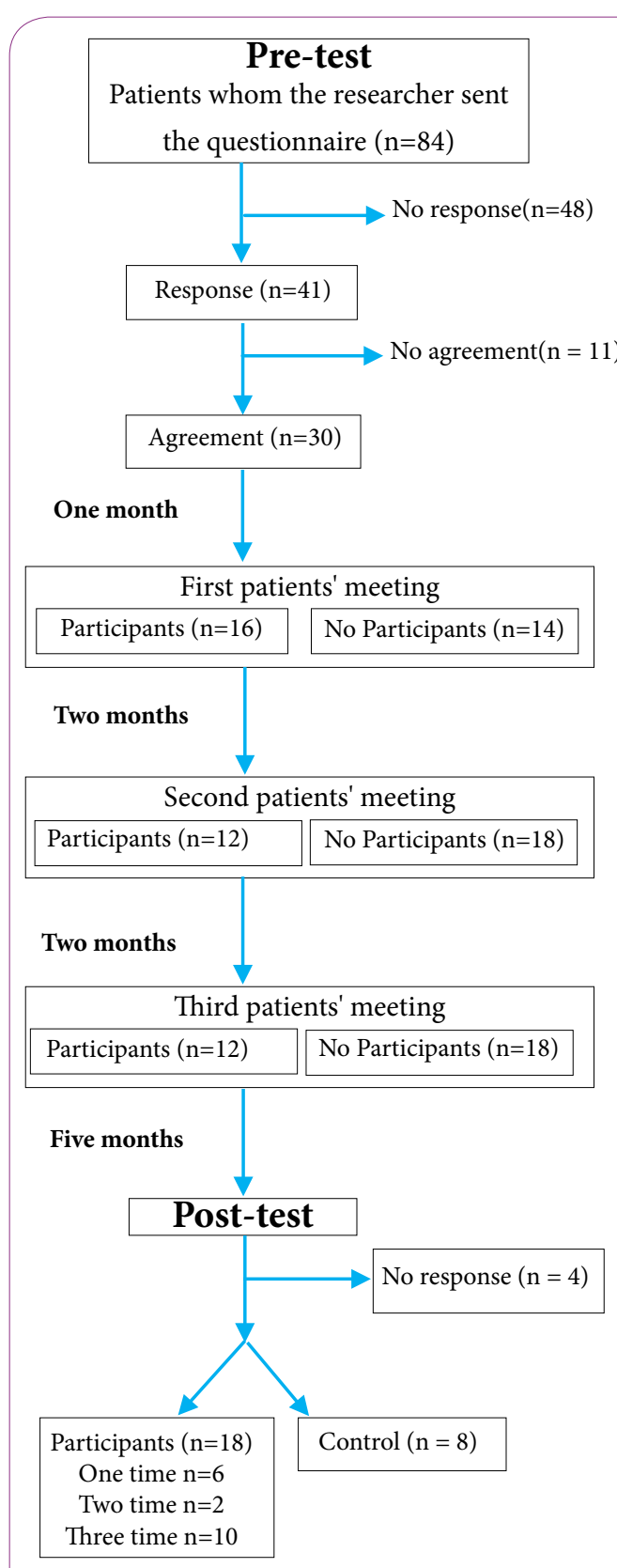

Figure 1: Overview of the entire design and participants in this study.

questionnaires of the participants. Anonymous questionnaire sheets were sent out one month before the First Meeting (pre-test) and five months after the Third Meeting (post-test). The participants' registration number was used to combine the two questionnaire sheets.

\section{Demographic data}

Demographic data were age, gender, date (year and month) of operation, chemotherapy, radiation therapy and diverting stoma.

\section{Knowledge and emotion}

Knowledge and Emotion were measured using visual analogue scale to verify practical effectiveness of the programs. Regarding Knowledge corresponding to Health Education, comprehension on defecatory dysfunction, coping with defecatory dysfunction, cancer 
and working life were evaluated. Regarding Emotions explored in group discussions, fear associated with cancer, feelings of loneliness, feelings of relief and confidence in daily life were evaluated [12]. The subjects marked their present condition incrementally on a $10-\mathrm{cm}$ line, with a higher number indicating a better condition.

\section{Defecatory dysfunction}

Defecatory dysfunction was measured using Defecation Dysfunction Assessment Scale ver.2 [20]. This scale is a selfadministered, validated and reliable instrument integrated for evaluating the defecatory dysfunction after surgery for colorectal cancer, and incorporates two functional domains (Fecal Continence and Passage, and Keeping Sense of Want to Do Defecate). A higher score indicates more difficulties in daily life.

\section{Quality of life}

Quality of life was evaluated by the European Organization for Research and Treatment of Cancer Quality of Life Questionnaire C30, version 3.0 (EORTC QLQ-C30). The EORTC has developed a cancer survivorship assessment which can be used as a stand-alone questionnaire. This questionnaire is a self-administrated, validated, and reliable instrument integrated for evaluating QOL in patients with cancer [21]. It comprises 30 questions and includes a global health and quality of life scale (global health status/QOL), five functional scales (physical, role, emotional, cognitive and social functioning), nine symptom scales (fatigue, nausea/vomiting, pain, dyspnea, insomnia, appetite loss, constipation, diarrhea, and financial difficulties). The scores of the scales range from 0 to 100 . A higher score on global health status/QOL and functional scales means higher QOL, but a higher score on the symptoms scales indicates more symptoms. Clinical significance of the changes corresponds to the following values: 5 to 9 is small; 10 to 19 is moderate; greater than 20 is large.

\section{Ethical considerations}

The researchers explained in writing the purpose of the study, data confidentiality and the assured privacy of participants, that it was voluntary participation, and that there was no disadvantage in not participating to the patient. The patients were asked to give their written consent. The study protocols were approved by the Ethical Committee of Wakayama Medical University (No. 1528).

\section{Data analyses}

To evaluate effectiveness of the patient group meetings, the subjects were divided into two categories: the Participant Group, which participated in the patients' meetings at least once $(n=18)$ and the Control Group, which did not participate in spite of having consented to the agreement $(\mathrm{n}=8)$.

The groups were compared by the demographic data of the pretest. Data are presented as the mean (standard deviation: SD) for continuous variables and as the percentage for discrete variables. $T$ test was used for continuous variables and Chi-squared or Fisher's exact test was used for discrete variables.

The statistical significance of changes in scores between the pretest and the post-test was evaluated by paired t test. The effect size (Cohen's $\mathrm{d}$ ) was calculated by dividing the difference between the pretest and post-test scores by the SD of the pre-test score. The effect size was interpreted as follows: $0.20-0.49$ as small, $0.50-0.79$ as moderate, and above 0.80 as large [22].
In order to determine the effectiveness of the patient group meetings, associations between the number of times that participants took part (attendance rate) and the variable were analyzed using multiple linear regression analysis (enter method), with the variable score at the post-test as the dependent variable, and with the attendance rate in the patient group meetings, variable score at the pre-test and age as independent variables.

Spearman's rank correlation was used to assess the relationships among changes in global health status/QOL, functional scales and symptom scales, and defecatory dysfunction.

Data were analyzed by SPSS version 23. A p value of $<0.05$ was considered statistically significant.

\section{Results}

\section{Participants}

The number of participants enrolled in the First Meeting was 16 out of 26 (participating ratio 62\%), 12 in the Second Meeting (46\%) and 12 in the Third Meeting (46\%) (Figure 1). Ten participants (38\%) attended all three meetings. Two (8\%) joined twice, and six (23\%) joined once. Eight (31\%) were not able to find the time to participate in the meetings, but answered the questionnaire sheets. Table 1 shows the demographic data of the subjects at the pre-test. There was no significant difference in items between the two groups.

\begin{tabular}{|c|c|c|c|c|}
\hline \multicolumn{2}{|l|}{ Group } & $\begin{array}{l}\text { Participant } \\
(\mathrm{n}=18)\end{array}$ & $\begin{array}{l}\text { Control } \\
(n=8)\end{array}$ & $\begin{array}{l}p \\
\text { value }\end{array}$ \\
\hline \multicolumn{2}{|l|}{ Age } & $65.2(7.5)$ & $66.8(7.6)$ & 0.673 \\
\hline \multicolumn{2}{|l|}{ Age at time of survey } & $39.4(8.7)$ & $41.0(11.4)$ & 1.000 \\
\hline & & $\mathrm{N}(\%)$ & $\mathrm{N}(\%)$ & \\
\hline \multirow[t]{2}{*}{ Gender } & Male & $15(83)$ & $6(75)$ & \multirow[t]{2}{*}{0.619} \\
\hline & Female & $3(17)$ & $2(25)$ & \\
\hline \multirow[t]{3}{*}{ Attendance rate } & 1time & $6(33)$ & 0 & \\
\hline & 2time & $2(11)$ & 0 & \\
\hline & 3time & $10(56)$ & 0 & \\
\hline \multirow[t]{3}{*}{ The year of surgery } & 2011 & $3(17)$ & $3(38)$ & \multirow[t]{3}{*}{0.452} \\
\hline & 2012 & $7(39)$ & $3(37)$ & \\
\hline & 2013 & $8(44)$ & $2(25)$ & \\
\hline \multirow[t]{2}{*}{ Chemotherapy } & Yes & $8(44)$ & $4(50)$ & \multirow[t]{2}{*}{1.000} \\
\hline & No & $10(56)$ & $4(50)$ & \\
\hline \multirow[t]{2}{*}{ Radiation therapy } & Yes & 0 & 0 & \multirow[t]{2}{*}{-} \\
\hline & No & $18(100)$ & $8(100)$ & \\
\hline \multirow[t]{2}{*}{ Diverting stoma } & Yes & $6(33)$ & $5(63)$ & \multirow[t]{2}{*}{1.000} \\
\hline & No & $12(67)$ & $3(37)$ & \\
\hline
\end{tabular}

Table 1: Demographic data of subjects $(n=26)$.

continuous variables: mean (standard deviation), Mann-Whitney $\mathrm{U}$ discrete variables: Chi-square. Fisher's exact test

\section{Changes in knowledge and emotion}

Table 2 shows the scores for Knowledge and Emotion of the subjects at the pre-test and at the post-test. Scores of four items in Knowledge in the Participant Group significantly increased in the post-test. Those in the Control Group showed no increase. 
Citation: Tsuji A, Nakayama M, Takifuji K, Yamaguchi M, Ueda I, et al. (2017) Effectiveness of Patient Group Meetings for Postoperative Patients with Defecatory Dysfunction. Int J Nurs Clin Pract 4: 260. doi: https://doi.org/10.15344/2394-4978/2017/260

Page 4 of 7

\begin{tabular}{|c|c|c|c|c|c|}
\hline & Group & Pre-test & Post-test & $\mathrm{p}$ value $\dagger$ & Effect size (d) \\
\hline \multirow[t]{2}{*}{ Comprehension on defecatory dysfunction } & Participant & $3.0(1.9)$ & $7.8(1.4)$ & .000 & 2.88 \\
\hline & Control & $3.3(3.1)$ & $4.1(2.8)$ & .115 & 0.27 \\
\hline \multirow[t]{2}{*}{ Coping with defecatory dysfunction } & Participants & $3.1(2.1)$ & $7.6(2.0)$ & .000 & 2.20 \\
\hline & Control & $2.3(3.1)$ & $2.9(2.9)$ & .118 & 0.20 \\
\hline \multirow[t]{2}{*}{ Information on cancer } & Participant & $3.7(2.0)$ & $6.9(2.2)$ & .000 & 1.60 \\
\hline & Control & $4.9(2.2)$ & $5.5(2.3)$ & .206 & 0.27 \\
\hline \multirow[t]{2}{*}{ Information on working life } & Participant & $3.3(2.7)$ & $6.1(2.9)$ & .005 & 1.00 \\
\hline & Control & $4.0(3.8)$ & $4.7(3.5)$ & .296 & 0.19 \\
\hline \multirow[t]{2}{*}{ Fear associated with cancer } & Participant & $5.4(3.7)$ & $6.7(3.1)$ & .162 & 0.38 \\
\hline & Control & $4.9(3.3)$ & $4.7(3.4)$ & .650 & 0.06 \\
\hline \multirow[t]{2}{*}{ A feeling of loneliness } & Participant & $4.7(3.6)$ & $5.5(3.5)$ & .290 & 0.23 \\
\hline & Control & $6.5(3.5)$ & $6.5(3.6)$ & 1.00 & 0.00 \\
\hline \multirow[t]{2}{*}{ A feeling of relief } & Participant & $4.7(3.1)$ & $7.3(1.8)$ & .005 & 1.03 \\
\hline & Control & $5.5(3.7)$ & $5.8(3.5)$ & .276 & 0.08 \\
\hline \multirow[t]{2}{*}{ Confidence about daily life } & Participant & $5.2(3.3)$ & $7.4(2.1)$ & .012 & 0.80 \\
\hline & Control & $5.8(3.5)$ & $6.3(3.1)$ & .356 & 0.15 \\
\hline
\end{tabular}

Table 2: Scores of in knowledge and emotion at pre-test and post-test.

Mean(Standard deviation)

tpaired t test

\begin{tabular}{|l|l|l|l|l|l|}
\hline & Group & Pre-test & Post-test & p value $\dagger$ \\
\hline $\begin{array}{l}\text { Fecal continence and passage } \\
(\text { range } 8-40)\end{array}$ & Participant & $23.8(7.4)$ & $22.2(7.3)$ & .150 \\
\cline { 2 - 6 } & Control & $23.9(7.9)$ & $23.8(6.4)$ & .947 & 0.22 \\
\hline $\begin{array}{l}\text { Keeping sense of want to do defecate } \\
(\text { range } 4-20)\end{array}$ & Participant & $12.1(3.2)$ & $11.4(3.0)$ & .209 & 0.01 \\
\cline { 2 - 6 } & Control & $10.8(4.0)$ & $11.5(3.2)$ & .222 & 0.14 \\
\hline
\end{tabular}

Table 3. Scores of defecatory dysfunction at pre-test and post-test.

Mean(Standard deviation)

tpaired $\mathrm{t}$ test

The scores of two items, "a feeling of relief" and "confidence about daily life" in Emotion in the Participant Group also significantly increased at the post-test. Those in the Control Group showed no increase.

\section{Changes in defecatory dysfunction}

Table 3 shows the score of defecatory dysfunction of the subjects at the pre-test and at the post-test. The changes in the scores between pre-test and post-test were not significant in either group or in either domain.

\section{Changes in QOL}

The scores of the post-test were compared with those of the pre-test The global health status/QOL clinically showed a small increase in the Participant Group, but the change was not significant (Table 4a). The changes in the Control Group showed no clinical significance.

Among the five Functional Scales (Table 4a), Social Functioning SF clinically showed moderate and significant increase in the Participant Group, but showed no clinical increase in the Control Group.

Among the nine Symptoms Scales (Table 4b), Diarrhea DI in the Participant Group significantly decreased. The scores in the Control
Group clinically increased in Fatigue FA, Dyspnea DY, Diarrhea DI and clinically there was a small decrease in Pain PA, Insomnia SL and Appetite loss AP, although the differences were not significant.

The factors significantly associated with the attendance rate in the patient group meetings are shown in Table 5. There were three factors in Knowledge, and two factors in the QOL. The standardized regression coefficients were positive in Knowledge and showed that the variable increased as the attendance rate increased. They were negative in Fatigue FA and Diarrhea DI, and showed that the variable decreased as the attendance rate increased.

\section{Changes in defecatory dysfunction}

The changes in global health status/QOL showed a significantly moderate negative correlation to those in Physical Functioning ( $\mathrm{r}=-$ .427, $\mathrm{p}=.029)$, Insomnia $(\mathrm{r}=-.458, \mathrm{p}=.019)$, and Diarrhea $(\mathrm{r}=-.465$, $\mathrm{p}=.017)$. Changes in Diarrhea were not related to those in Fatigue but correlated to those in the global health status/QOL $(\mathrm{r}=-.465, \mathrm{p}=.017)$ and Social Functioning $(\mathrm{r}=-.505, \mathrm{p}=.008)$. Changes in Fatigue showed a significantly negative correlation to those in Role Functioning $(\mathrm{r}=-.472, \mathrm{p}=.015)$ and Cognitive Functioning $(\mathrm{r}=-.648, \mathrm{p}=<0.001)$. Changes in scores of the two domains of Defecatory Dysfunction were not related to scales of global health status/QOL and QOL in Functional and Symptoms scales. 
Citation: Tsuji A, Nakayama M, Takifuji K, Yamaguchi M, Ueda I, et al. (2017) Effectiveness of Patient Group Meetings for Postoperative Patients with Defecatory Dysfunction. Int J Nurs Clin Pract 4: 260. doi: https://doi.org/10.15344/2394-4978/2017/260

Page 5 of 7

\begin{tabular}{|l|l|l|l|l|l|}
\hline \multirow{2}{*}{ QOL } & Group & Pre-test & Post-test & p value $\dagger$ & Effect size (d) \\
\cline { 2 - 6 } & Conticipant & $71.3(20.3)$ & $78.1(17.4)$ & .208 & 0.40 \\
\hline \multirow{3}{*}{ PF2 } & Participant & $91.8(17.8)$ & $70.9(13.4)$ & .851 & 0.06 \\
\cline { 2 - 6 } & Control & $90.9(12.2)$ & $89.5(15.2)$ & .683 & 0.10 \\
\hline \multirow{3}{*}{ RF2 } & Participant & $85.2(16.9)$ & $86.2(15.3)$ & .785 & 0.06 \\
\cline { 2 - 6 } & Control & $73.0(21.8)$ & $71.0(19.3)$ & .613 & 0.10 \\
\hline \multirow{3}{*}{ FF } & Participant & $90.0(12.6)$ & $88.9(17.0)$ & .828 & 0.07 \\
\cline { 2 - 6 } & Control & $75.0(17.0)$ & $77.3(13.1)$ & .569 & 0.15 \\
\hline \multirow{3}{*}{ CF } & Participant & $81.5(15.9)$ & $83.3(15.0)$ & .552 & 0.12 \\
\cline { 2 - 6 } & Control & $70.8(33.0)$ & $68.8(13.7)$ & .857 & 0.08 \\
\hline \multirow{3}{*}{ SF } & Participant & $84.2(20.2)$ & $94.4(11.4)$ & .017 & 0.62 \\
\cline { 2 - 6 } & Control & $85.5(20.7)$ & $87.6(17.1)$ & .797 & 0.11 \\
\hline
\end{tabular}

Table 4a: Scores of QOL at pre-test and post-test: global health status/ QOL, five functional scales

QOL: Global health status, PF2: Physical functioning, RF2: Role functioning, EF: Emotional funcyioning, CF: Cognitive functioning, SF: Social functioning Mean (Standard deviation)

tpaired $t$ test

\begin{tabular}{|l|l|l|l|l|l|}
\hline & Group & Pre-test & Post-test & p value $\dagger$ & Effect size (d) \\
\hline \multirow{3}{*}{ FA } & Participant & $13.8(13.8)$ & $15.3(13.7)$ & .626 & 0.11 \\
\cline { 2 - 6 } & Control & $22.0(18.4)$ & $33.0(15.8)$ & .134 & 0.64 \\
\hline \multirow{3}{*}{ NV } & Participant & 0.0 & $1.83(7.8)$ & .331 & 0.33 \\
\cline { 2 - 6 } & Control & 0.0 & 0.0 & - & - \\
\hline \multirow{3}{*}{ PA } & Participant & $10.2(12.9)$ & $14.8(19.7)$ & .392 & 0.28 \\
\cline { 2 - 7 } & Control & $16.6(19.8)$ & $10.4(15.1)$ & .080 & 0.35 \\
\hline \multirow{3}{*}{ DY } & Participant & $11.0(16.0)$ & $11.0(16.0)$ & 1.000 & 0.00 \\
\cline { 2 - 6 } & Control & $4.1(11.7)$ & $12.4(17.1)$ & .170 & 0.57 \\
\hline \multirow{3}{*}{ SL } & Participant & $16.5(20.4)$ & $17.4(16.5)$ & .883 & 0.05 \\
\cline { 2 - 6 } & Control & $33.1(35.5)$ & $24.8(23.3)$ & .351 & 0.28 \\
\hline \multirow{3}{*}{ AP } & Participant & 0.0 & $1.83(7.8)$ & .331 & 0.33 \\
\cline { 2 - 6 } & Control & $24.9(34.4)$ & $12.4(17.1)$ & .401 & 0.46 \\
\hline \multirow{3}{*}{ CO } & Participant & $25.7(24.2)$ & $27.5(25.9)$ & .772 & 0.07 \\
\cline { 2 - 6 } & Control & $37.3(33.0)$ & $41.3(23.3)$ & .741 & 0.14 \\
\hline \multirow{2}{*}{ DI } & Participant & $23.8(19.0)$ & $14.7(16.9)$ & .020 & 0.51 \\
\cline { 2 - 6 } & Control & $24.8(23.3)$ & $33.0(24.9)$ & .170 & 0.34 \\
\hline \multirow{3}{*}{ FI } & Participant & $16.5(23.3)$ & $12.8(16.6)$ & .542 & 0.18 \\
\cline { 2 - 6 } & Control & $16.5(24.9)$ & $16.5(17.6)$ & 1.000 & 0.00 \\
\hline
\end{tabular}

Table 4b: Scores of QOL at pre-test and post-test: nine symptoms scales.

FA: Fatigue, NV: Nausea and vomiting, PA: Pain, DY: Dyspnoea, SL: Insomnia, AP: Appetite loss, CO: Constipation, DI: Diarrhoea, FI: Financial difficulties

Mean (Standard deviation)

tpaired $t$ test

\section{Discussion}

The patient group meeting is one of the support group interventions. Such support group intervention for breast cancer patients has received attention $[13,14,17]$ and its effectiveness has been evaluated by Knowledge and QOL [18], the Hospital Anxiety and Depression Scale [18, 19], Profile of Mood States[19] and EORTIC QLQ-30 [13]. The support group intervention on chronic diseases related to cancer has not been investigated.

In this study, participants in the patient group meetings gained information on defecatory dysfunction and coping with diarrhea.

\begin{tabular}{|l|l|l|l|}
\hline \multicolumn{2}{|l|}{} & $\begin{array}{l}\text { Standardized } \\
\text { regression } \\
\text { coefficient }\end{array}$ & $\mathrm{p}$ value \\
\hline $\begin{array}{l}\text { Knowledge } \\
\text { and emotion }\end{array}$ & $\begin{array}{l}\text { Comprehension on } \\
\text { defecatory dysfunction }\end{array}$ & .424 & 0.034 \\
\cline { 2 - 4 } & $\begin{array}{l}\text { Coping with defecatory } \\
\text { dysfunction }\end{array}$ & .558 & 0.002 \\
\cline { 2 - 4 } & Information on cancer & .458 & 0.012 \\
\hline \multirow{2}{*}{ QOL } & FA & -.533 & 0.001 \\
\cline { 2 - 4 } & DI & -.432 & 0.003 \\
\hline
\end{tabular}

Table 5: Factors significantly associated with the attendance rate in the patient group meeting.

\section{multiple linear regression analysis (enter method)}

variable score at post-test as the dependent variable

the attendance rate in the patient group meeting, variable score at pre-test and age as

independent variables

FA: Fatigue, DI: Diarrhoea

Mean (Standard deviation)

tpaired $t$ test

They reported higher global health status/QOL due to lower diarrhea symptoms. A decreased symptom score in Diarrhea was related tothe increase in Social Functioning. This change was associated with the attendance rate in the patient group meetings. These results, therefore, suggest the effectiveness of the patient group meetings. To our knowledge, this is the first study to clarify the efficacy of the patient group meetings for LAR postoperative patients using knowledge, emotion and QOL of such patients.

Participants of the patient group meetings gained information on coping with diarrhea, which - among the various symptoms - has a severe effect on daily life due to defecation dysfunction, and acquired ways with which to cope with the diarrhea by themselves. They shared their experiences with other patients dealing with the same disease. The interaction facilitated a reduction in participants' anxiety, provided them with a feeling of relief, and helped to create a positive mood in daily life.

In an earlier study, the scores of Diarrhea of patients after LAR or high anterior resection in the same evaluation instrument were 30.7 before operation, 12.2 at six months and 9.3 at 12 months after surgery [23]. Compared with this earlier report, the score of Diarrhea in this study was higher. This implies that the participants in this study had been more prone to worry about diarrhea.

The difference in score of Diarrhea correlates to that of global health status/QOL and Social Functioning but does not correlate to scores of defecatory dysfunction. The change in score of Diarrhea may not have resulted from an improvement in diarrhea symptoms due to better defecatory function. On the contrary, we can postulate that the patients' participation in the patient group meetings, where they gained knowledge on defecatory dysfunction and ways with which to cope with it, brought about the improved score for Diarrhea. Becoming able to cope with diarrhea may, in all likelihood, have given rise to higher global health status/QOL and Social Functioning.

The Participant Group was at the same fatigue state as the pre-test, but those in the Control Group tended to exhibit compounded fatigue symptoms at the post-test. These conditions could very well have underscored a relationship with the patients' attendance rate in, or absence from, the meetings. That is, the increased fatigue symptoms could have prevented some patients in the Control Group from taking part in these meetings. It is, therefore, also necessary to seek out the 
Citation: Tsuji A, Nakayama M, Takifuji K, Yamaguchi M, Ueda I, et al. (2017) Effectiveness of Patient Group Meetings for Postoperative Patients with Defecatory Dysfunction. Int J Nurs Clin Pract 4: 260. doi: https://doi.org/10.15344/2394-4978/2017/260

Page 6 of 7

source of fatigue and provide the patients with support needed to alleviate it.

The patient group meeting intervention is effective for increasing the QOL of patients who combat cancer and associated problems over a long period time. To make it more effective, timing and contents of the program need to be studied and defined.

In our study, diarrhea symptoms continued for one year and nine months after surgery [12]. It is, for this reason, preferable to have the patients participate in the group meetings while dealing with the symptoms, ideally for two years after the operation.

Earlier research has already shown that peer support between cancer survivors fosters supportive intercommunication, empowers the patient, and, further, introduces positive effects on psychosocial function and QOL [24]. Programs especially tailored for patients conditions are evidently effective in building positive QOL. We strongly recommend, therefore, that in addition to a peer support program, programs for patients with defecatory dysfunction include psychoeducation for coping with the symptoms to assist them in managing their symptoms.

Possible limitations of this study should be mentioned. The sample size was small. Although the observation period was not short, there were few assessments over a long period of time. Small number of participants prevented from the three-time evaluation of patient group meeting. Accordingly, the results cannot be generalized to the entire LAR postoperative patient population suffering with defecatory dysfunction. However, this study provides a start for finding the means with which to provide adequate care for patient's QOL.

\section{Conclusions}

The patient group meetings were held three times to clarify the effectiveness on QOL of the LAR postoperative patients with defecatory dysfunction. In the symptoms scales of QOL, measured by EORTC QLQ-C30, the score of Diarrhea significantly decreased concomitantly with the attendance rate, and was independent of defecatory dysfunction. Therefore, the patient group meetings certainly appear to have provided positive health effects on survival related to QOL. Further research needs be carried out with larger samples in order to develop methods that enable case-by-case, longlasting support for such patients.

\section{Competing Interests}

The authors declare that they have no competing interests.

\section{Author Contributions}

AT, MN, KT, IM, YS, and KM were responsibility for study design. AT, MN, KT, MY, IU and YS were for data collection. AT, MN, KT, IM, YS and KM were for analysis.AT, MN, KT, MY, IU, IM, YS and KMwere for manuscript preparation.

\section{Acknowledgments}

The authors thank the study respondents for answering the questionnaire and participants in the patients' meeting for participating at various times and sharing their golden experiences.

\section{Funding}

Thanks to JSPS KAKENHI Grant Number JP15K11633 for the financial support.

\section{References}

1. Center for Cancer Control and Information Services, National Cancer Center. http://ganjoho.jp/public/index.html. (accessed 20 October 2017)

2. Di Betta E, D’hoore A, Filez L, Penninckx F(2003) Sphincter saving rectum resection is the standard procedure for low rectal cancer. Int $\mathrm{J}$ Colorectal Dis 18:463-469.

3. Ekkarat P, Boonpipattanapong T, Tantiphlachiva K, Sangkhathat S (2016) Factors determining low anterior resection syndrome after rectal cancer resection: A study in Thai patients. Asian J Surg 39: 225-231.

4. Ziv Y, Zbar A, Bar-Shavit Y, Igov I (2013) Low anterior resection syndrome (LARS): cause and effect and reconstructive consideration. Tech Coloproctol17: 151-162

5. Matsuda K, Hotta T, Takifuji K, Yokoyama S, Oku Y, et al. (2015) Randomized clinical trial of defaecatory function after anterior resection for rectal cancer with high versus low ligation of the inferior mesenteric artery. Br J Surg 102: 501-508

6. Landers M, McCarthy G, Savage E (2012) Bowel symptom experiences and management following sphincter saving surgery for rectal cancer: A qualitative perspective. Eur J Oncol Nurs16: 293-300.

7. Desnoo L, Faithfull SL (2006) A qualitative study of anterior resection syndrome: the experiences of cancer survivors who have undergone resection surgery. Eur J Cancer Care (Engl)15: 244-251.

8. Scheele J, Lemke J, Meier M, Sander S, Henne-Bruns D, et al. (2015) Quality of life after sphincter-preserving rectal cancer resection. Clin Colorectal Cancer 14: e33-40.

9. Maslyankov S, Penchev D, Todorov G, Vladov N (2015) A meta-analysis of quality of life, estimated by questionnaires of the European Organization for Research and Treatment of Cancer (EORTC) after rectal cancer surgery. Chirurgia (Bucur) 110: 356-361.

10. Juul T, Ahlberg M, Biondo S, Espin E, Jimenez LM, et al. (2014) Low anterior resection syndrome and quality of life: An international multicenter study. Dis Colon Rectum 57:585-591.

11. Cornish JA, Tilney HS, Heriot AG, Lavery IC, Fazio VW, et al. (2007) A meta-analysis of quality of life for abdominoperineal excision of rectum versus anterior resection for rectal cancer. Ann Surg Oncol 14: 2056-2068.

12. Tsuji A, Suzuki Y, Morioka I (2015) Clues to supporting a patient who underwent a low anterior resection operation. Int J Nurs Clin 2: 129

13. Björneklett HG, Rosenblad A, Lindemalm C, Ojutkangas $M L$, Letocha $H$, et al. (2013) Long-term follow-up of a randomized study of support group intervention in women with primary breast cancer. J Psychosom Res 74: 346-353.

14. Coward DD (2003) Facilitation of self-transcendence in a breast cancer support group: II. Oncol Nurs Forum 30: 291-300.

15. Johnson J (1982) The effects of a patient education course on persons with a chronic illness. Cancer Nurs 5: 117-123.

16. Fawzy FI, Canada AL, Fawzy NW (2003) Malignant melanoma: effects of a brief, structured psychiatric intervention on survival and recurrence at 10 year follow-up. Arch Gen Psychiatry 60:100-103.

17. Fawzy FI, Cousins N, Fawzy NW, Kemeny ME, Elashoff R, et al. (1990) A structured psychiatric intervention for cancer patients. I. Changes over time in methods of coping and affective disturbance. Arch Gen Psychiatry 47: 720-725.

18. Fukui S, Kugaya A, Kamiya M, Koike M, Okamura H, et al. (2001) Participation in psychosocial group intervention among Japanese women with primary breast cancer and its associated factors. Psychooncology 10: 419-427. 
Citation: Tsuji A, Nakayama M, Takifuji K, Yamaguchi M, Ueda I, et al. (2017) Effectiveness of Patient Group Meetings for Postoperative Patients with Defecatory Dysfunction. Int J Nurs Clin Pract 4: 260. doi: https://doi.org/10.15344/2394-4978/2017/260

19. Fukui S, Kugaya A, Okamura H, Kamiya M, Koike M, et al. (2000) A psychosocial group intervention for Japanese women with primary breast carcinoma. Cancer 89: 1026-1036.

20. Sato M (2010) Development of "Defecatory Dysfunction Assessment Scale ver.2" for evaluation after anterior resection for rectal cancer. J Jpn Soc Stoma Continence Rehab 26: 37-48.

21. Aaronson NK, Ahmedzai S, Bergman B, Bullinger M, Cull A, et al. (1993) The European Organization for Research and Treatment of Cancer QLQ-C30: a quality-of-life instrument for use in international clinical trials in oncology. J Natl Cancer Inst 85: 365-376.

22. Cohen J (1992) A power primer. Psychol Bull 112:155-159.

23. Tsunoda T, Nakao N, Watanabe M (2011) Comparison of the quality of life in patients undergoing low anterior resection or high anterior resection for rectal cancer. Jpn J Gastroenterol Surg.44: 795-802.

24. Mcmullen C, Liu L, Bulkley JE, Hornbrook MC, Wendel C, et al. (2017) Participation in activities associated with quality of life for long-term survivors of rectal cancer. Perm J 21: 48-54. 